\author{
Giorgio Maria Paolo Graziano ${ }^{1}$ Marco \\ Cavallaro $^{2}$ and Antonino Graziano ${ }^{3 *}$ \\ ${ }^{1}$ University of Catania, Medical School, Italy \\ ${ }^{2}$ Radiology unit ASP Ragusa, Italy \\ ${ }^{3}$ University of Catania, Azienda Policlinico Dpt \\ Sciences Surgery and advanced technologies, " $G$ \\ Ingrassia", via S Sofia 86, Catania, Italy
}

Dates: Received: 16 November, 2015; Accepted:

09 January, 2016; Published: 19 January, 2016

*Corresponding author: Prof. Graziano Antonino, via s Sofia n 87 Catania, cap 95125 Sicilia Italy, University of Catania, Italy, Tel: 0953782880; E-mail: graziano@policlinico.unict.it

www.peertechz.com

ISSN: 2455-2968

Keywords: Hereditary familial adenomatous polyposis treatment

\section{Research Article \\ The Familial Adenomatous Polyposis. A Difficult Problem, Between Prevention and Treatment}

\begin{abstract}
Introduction: The familial adenomatous polyposis of the colon (FAP) is a rare hereditary disease transmitted as an autosomal dominant trait due to the mutation of the APC gene. The purpose of the study is to assess, even according to the data of our experience, the possibility of clinical application of the results of the new genetic research, linking the problem to prevention and/or treatment. Some operative remarks conclude the study.
\end{abstract}

Materials and methods: We observed (II clinical surgery Garibaldi Hospital in Catania, III Surgery, and Digestive Surgery Unit of the University of Catania) 16 patients on all. In Group I (FAP + ) were observed 12 patients with FAP (5 male and 7 female). In group II (FAP-) 4 cases (male). All patients were part of No. 6 families. The diagnostic test and colonoscopy were diagnostics. 8 patients (and all their relatives), performed the genetic typing.

Results: The ileo-anal anastomosis with reservoir (IPAA), was, time to time, the operation chosen especially in cases of degeneration in the rectal tract. The ileo-rectal anastomosis (IRA) has been used mostly in the prevention phase of degeneration. The surgical treatment involved 12 patients (Group I). 7 of the cases treated, were subjected to a procedure proctocolectomy and reconstruction by J-pouch ileo-anal anastomosis or latero-terminal ileo-anal anastomosis, with temporary protective ileostomy. One patient underwent definitive ileostomy after proctocolectomy because of cancer involving the ano-pettinea line. The other 4 were subjected to IRA (3), with ileo-rectal anastomosis to $8-10 \mathrm{~cm}$. A patient has developed a pelvic abscess, which required reoperation the definitive iliac anus. Mortality was absent. In the remaining 4 cases of Group II, the genetic test proposed reveal not mutations of the APC gene, so the presumed late degeneration of the polyp did opt for watchful waiting

Discussion: The therapeutic approach in FAP is particularly complex and challenging, because it includes the prophylaxis and treatment of the adenomas, for colorectal cancer and ECD (extra colonic diseases) dangerous according to their severity and location. In practice, the FAP has problems similar to the treatment of recto-ulcerative colitis (UC). The indication to anoproctocolectomia with definitive ileostomy can be offered only to those patients (1 case in our series) in which there is an invasive rectal cancer too close or involving the anal rhyme, with diffuse polyposis of the colon. In other cases ( 2 in our experience one in the rectum, and one in the recto-sigma junction) of cancerous evolution, and when the rectum is intensely involved from polyps (5 of our patients) is preferable to the IPAA and ileo pouch anal anastomosis in $\mathrm{J}$, . In all cases where it is considered possible (4 cases came under our observation) the endoscopic procedure of the rectum and there is no cancer in situ, you can proceed to a total colectomy with ileo-rectum anastomosis about $6 \mathrm{~cm}$ or below to achieve good continence. It is advisable to carry out an T-L ileo-rectal anastomosisor with aJ pouch in cases where it is deemed necessary to remove of the proximal rectum with more intensely polyposis. A number of evacuations acceptable (up to 3-5 per day) are the functional results of these predictable surgical options. Dehiscence with sepsis of the small pelvis may complicate the postoperative evolution of IPAA, which can be controlled with a protective ileostomy. He IRA involves especially the risk of degeneration of the residual or recurrent rectal polyps, while the IPAA generally results in imperfect functional results, risk of dehiscence and septic complications

Conclusion: The FAP is a rare but potentially lethal disease (responsible for about $1 \%$ of all colic cancers, HPCC) for the patient, in which a genetic mutation is transmitted in $50 \%$ of the descendants. The introduction into clinical practice of test for molecular diagnosis is the innovation technology, which allows a more accurate assessment of the disease and the best indications of the time and type of treatment and surveillance program. , the prospects of Genetics for further more direct approaches, present choices ethically more complex of prevention and treatment.

\section{Indroduction}

The familial adenomatous polyposis of the colon $(\mathrm{FAP}){ }^{\star}$ is a rare hereditary disease, transmitted as an autosomic dominant trait due to the mutation of the APC gene ${ }^{\star *}$, characterized by the appearance of a high number of adenomatous polyps in the colon mucosa $(>100-$ 1000 and over) In 1925 Lockhart-Mummery focused his attention on a particular characteristic of this disease and on his condition of almost mandatory precancerosis that in the short space of $10-15$ years after the onset of polyps develop with certainty a cancer (BC Morson, 
T . Kozuka) [1-4]. Subsequent studies (C. Dukes, HJR Bussey) [5], have quantified the risk of degeneration in relation to age, size of the polyps, histological type, degree of dysplasia. In view of the malignant potential of the disease, surgical therapy has always been addressed to a principle of radical demolition, even at the stage of simple polyposis, and even with the risk of unsatisfactory functional results. However, since a considerable number of patients is subjected to interventions at an early age with a high need for productive and sexual activity and with a long lease of life remaining, the problems of continence and thus the quality of life must be held in high esteem and perhaps address towards solutions of compromise between radicalism and continence, at least in the phase of simple dysplasia. The purpose of the study is to assess, also based on the results of our experience, the impact that the new diagnostic possibilities offered by genetics can have on the screen, on the monitoring and treatment of the disease. Much over, between prevention and treatment to driving operative remarks of proceeding [6-8]. The role of heredity in colorectal carcinoma (RCC) is evident in the polyposis syndromes HPCC (FAP, with variants of Gardner and Turcot, rich in ECD, the AFAP and MAP) and especially in syndromes HNPCC (S. Lynch), ie the variants of polyposis or non-polyposis hereditary colorectal cancer. The FAP is characterized by the onset, early or late in 'childhood or adolescence, of hundreds or thousands of polyps (still more than 100) on which sooner or later develops a malignancy [8-10] Inheritance is autosomal dominant and the prevalence is $1 / 8000$ individuals. The HPCC carcinoma represents $1 \%$ of all colorectal carcinomas, while the HNPCC about 6-8\%. Although colectomy and especially procto-colectomy can considerably reduce up to zero the risk of $\mathrm{CCR}$, the disease requires frequent endoscopic surveillance, genetic typing and, in some variants, is associated with other diseases such as tumors and dermoid cysts (itself more therapeutic problems for the site and size that can reach), osteomas, pigmented hypertrophy of the retina, thyroid tumors, carcinomas of the duodenum and small intestine, hepatoblastomas and tumors of the CNS. The FAP is produced by mutations in the APC gene, in the locus $5 q$ 21-22, 8538 bp long, consisting of 16 exons, coding for a protein of the type of beta catenin involved in the preservation of the contacts between epithelial cells and in the regulating their proliferation, through action on the cell cycle and apoptosis. Since 1994 there is a genetic test that has modified the guidelines allowing the disease to exclude from followup family members who do not carry congenital alteration, although it is seen that may come into play other polyposis mutations. In FAP are described over 300 mutations of the gene. Those of the first four exons are correlated with the phenotype AFAP. The hypertrophic chorio-retinitis is characteristic of the mutations of exons 10-15. Mutations between codons 1400 and 1600 are associated with extracolonic localizations. Most interesting are alterations of codons 1250 and 1464, associated with increased risk for cancer of the rectal stump and alterations of codon 1309, related with the early onset of the disease (C. Dodaro: topical theme of family colonic polyposis, 2010). The AFAP is a condition in which patients are carriers of a few polyps, less than 100. The disease is caused by mutations at the 3' or 5' end of the APC gene or of certain areas of exon 9. The disease is defined as AFAP (attenuated FAP). In these cases, the CRC may arise later in life, are less common extra-intestinal lesions and the disease can appear sporadically. Have been described patients with a clinical picture similar to the above, even in the absence of a multi-generational history of polyposis and in the absence of alterations of the APC gene. It should be noted that mutations may occur in somatic cells and are therefore not to be heritable, but in the specific case there are important germline mutations. In these patients, typically 30 - 60 years old, with multiple polyps but no APC alterations can be detected a change in the gene MutYH (MYH) and the disease is called MAP. The gene in question [11], a BER (base excision repair), corrects the DNA damage caused by reactive oxygen species (ROS: super oxide ions, hydroxyl ions, etc.), chemical agents or ionizing radiation. From 23 to $30 \%$ of patients with more than 10 but less than 100 polyps are carriers of abiallelic mutation of $\mathrm{MYH}$ gene, which then is recessive, in the absence of inherited mutations of the APC gene. According to some, up to $2.8 \%$ of early CCR, could be ascribed to biallelic mutations (Y175C and G382D) of MYH gene, which means not only that these mutants may have the same clinical stigmata of FAP, but also reach out to colorectal cancer in the same if not greater extent. Genetic testing, to day available, should be reserved: a) to patients with clinical picture of FAP or AFAP when it's not demonstrated PCA gene alteration; $b$ ) to those cases of CCR with a pattern of recessive inheritance or multiple adenomas; c) to the children of known carriers of MYH mutations, d) to partners of known carriers of MYH mutations (may themselves be carriers), with the risk, if monoallelic, to transmit the disease in $50 \%$ of the offspring. According to Jenkins (2006), the cumulative risk for cancer at age of 70 for MYH mutation carriers is $8 \%$ (4-19\%) if monoallelic and $80 \%(35-100 \%)$ if biallelic. Therefore they should be treated the same way as the mutant APC, with colonoscopies and colectomy when indicated. However, being the later the age of onset of polyps and the risk of cancerous, endoscopic surveillance can begin at a later age, after 25 years. In patients with heredo-familiar colorectal cancer, but adverse for changes in MMR (HNPCC), shall still be looking for the alterations of genes APC and MYH, which in any case should be investigated in patients with more than one adenoma of the colon. Endoscopic and genetic screening is also recommended in relatives of first degree of biallelic carriers, particularly in the brothers who have a $25 \%$ risk of having mutations. The children biallelic mutants are still carriers of at least one mutation, if the partner is not a carrier. It is therefore reasonable to perform a base colonoscopy in these individuals and, if polyps are noticed, there will establish a surveillance program. The cornerstone of the treatment of colorectal polyps are celecoxib (seems that, for some authors, to reduce the number and size of polyps); endoscopic polypectomy, when possible, for AFAP and MAP is the first approach; the surgical option (colectomy and proctocolectomy), certainly in FAP characterized by a myriad of polyps, and in the already developed HPCC $[7,8]$.

${ }^{\star}$ Attenuated Familial Polyposis. ${ }^{* *}$ Adenomatous Polyposis Coli” mutation

\section{Materials and Methods}

The case study refers to 16 patients observed, of which 12 surgically treated at the Surgery Clinic II from 1986 to1994, at the III Surgery unit from 1996 to 2004 and from 1996 to 2013 at the digestive system surgery unit of University of Catania, Azienda Policlinico. In Group 
I (FAP positive) have been cataloged 12 FAP patients * of which $\mathrm{N}$ 7 women en 5 males. In group II were observed in 4 cases of male (100\%), suffering from multiple polyps of the colon, but negative for gene mutations FAP. All patients were part of No. 6 families in which most of the children had the FAP *. Adenomatous polyposis interested the entire colon and rectum in number $>100$ cases FAP; multiple polyps in the small number were found in the other cases, FAP negative (Figures 1-3).

The symptoms were related to diarrhea, bleeding, mucous excess in the stool and anemia in diffuse polyposis. There were adenomatous polyps in the rectum even at $2-3 \mathrm{~cm}$ from or on the anal verge. The diagnostic test of choice was colonoscopy biopsy which in 3 cases

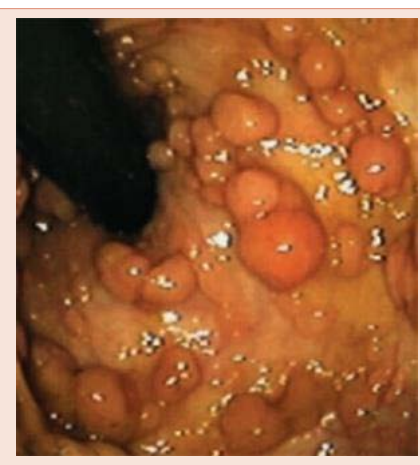

Figure 1: FAP *. Adenomatous polyposis the entire colon and rectum.

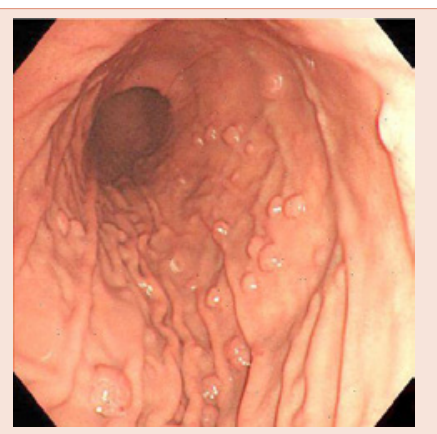

Figure 2: FAP *. Adenomatous polyposis the entire colon and rectum.

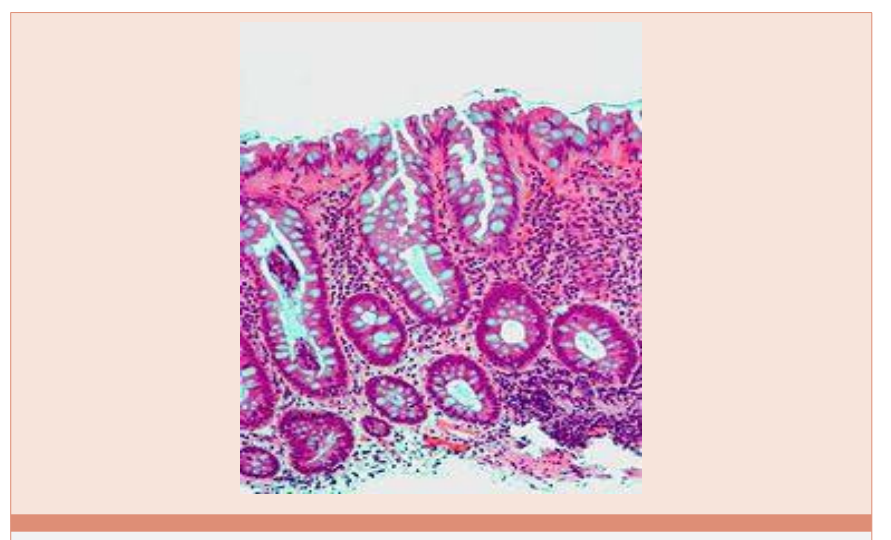

Figure 3: Histological cancer aspects. showed the cancer development on polyps in the rectum and sigmoid. The execution of TC and the dosage of tumor markers of patients, completed the study CRC for the purpose of TNM staging. Have not been identified in the cases observed ECM to occur in other segments of the digestive tract or other organs. In patients CRC and other observed after the 90 's, DNA testing has identified mutations of the long arm of chromosome 5 localized in the gene APC and MYH gene alterations ${ }^{* * *}$. Therefore, the treatment has been diversified in time.

\section{*** Gene mutations}

\section{Results}

In three of the cases observed is documented, with a histological diagnosis on endoscopic biopsies, a carcinomatous degeneration (case 1 behind the pectineal line, case 2 in the rectum, case 3 in rectosigmoid junction).Stadium: pT1-2N0M0. The total ano-proctocolectomy as Miles was practiced in case 1 (rectal sphincter involved at examination of the piece removed). In the remaining cases 2 and 3 , has been practiced proctocolectomy with mucosectomy of the last 5 $\mathrm{cm}$ of the rectum and reconstruction by J -pouch (cases 2 ) or by sideterminal anastomosis (case 3) and temporary ileostomy. Mortality was absent. Also in other 5 cases of diffuse disease, with extensive involvement of the rectum, even in the absence of concretization in place, we opted for the IPAA. In one case there was an anastomotic pouch leak with pelvic sepsis, drained and settled with burrowing wizard. Stenosis at a distance of 40 days after surgery was the result of subclinical dehiscence. The stenosis was treated successively with progressive dilatation. The ileostomy was closed on average after 2 months after surgery with progressive functional restoration. The ability of the pouch was assessed with final radiological control. The cells in 4 cases at a distance of 1 month from the close of the ileostomy has stabilized in three daily bowel movements, in 4 cases persisted with 4-6 daily bowel movements for which it was required prolonged treatment ( 3 month) with anti-diarrheal drugs needed to adaptation. In the remaining four of the 12 cases of FAP positive polyposis not excessively involving the rectum, has led to use an ileum-rectal anastomosis (IRA) with endoscopic drainage and scheduled monitoring of the rectum, so far without concretization founding in polyps, except in a case of polyp cancer removed by endoscopy without base involving. In some cases, when the portion of the rectum to be removed was more consistent, it pushed, for the ileorectal anastomosis, to adopt or J pouch or side-terminal anastomosis, to improve continence. In 4 of the 16 cases observed as FAP negative resulting, the genetic test has led to a wait and go, although vigilant. In two of these cases resulting MYH mutants, endoscopic surveillance was complemented by treatment with COX2. Mutational analysis therefore plays an essential role in determining the age at which patients undergo surgical treatment and prophylaxis. In the last 4 patients it was therefore decided to postponedemolition therapy. The Table 1 summarizes the surgical treatment implemented.

\section{Discussion}

\section{Screening, treatment and surveillance}

Disease screening today is based, in families, on the search for genetic alteration (DNA testing) and on the examination of the fundus oculi that, in cases positive for FAP, requires endoscopic examination 
Table 1: Treatment surgery.

In Group I (FAP positive) have been cataloged 12 FAP patientsof which $\mathrm{N} 7$ women en 5 males

In group II were observed $n 4$ cases of male (100\%), suffering from multiple polyps of the colon, but negative for gene mutations FAP.

Histological group: FAP positive

$\mathrm{N} 3$ cases polyps carcinomatous degeneration (case 1: behind the pectineal line, case 2: in the rectum,case 3: in recto-sigmoid junction).Stadium: pT1-2N0M0.

the 5 cases of diffuse disease, with extensive involvement of the rectum, even in the absence of cancerization in place, :stadium pT3 NxMx(N 4 cases persisted with 4-6 daily bowel movements for which it was required prolonged treatment (3 month)

N4 cases stadium pT3 NxMx

FAP: negative:

N 4 cases of male (100\%), suffering from multiple polyps of the colon, but negative for gene mutations polyposis

Treatment surgical

FAP positive

A) diffuse diseasewe opted for the IPAA

the total ano-procto-colectomy as Miles was practicedin case 1: (rectal sphincter involved at examination of the piece removed).cases 2 and 3 , has been practiced proctocolectomy with mucosectomy of the last $5 \mathrm{~cm}$ of the rectum and reconstruction by $\mathrm{J}$-pouch (cases 2 ) or by side-terminal anastomosis (case 3 ) and temporary ileostomy a distance of 1 month from the close of the ileostomy,

B) ileum-rectal anastomosis (IRA)

FAP negative

In the last 4 patients it was therefore decided to postponedemolitiontherapy the genetic test has led to a wait and go, although vigilant. In two of these cases resulting MYH mutants, endoscopic surveillance was complemented by treatment with COX2

in adolescence, the later in the cases of negativity. Discussed is the repetition frequency of the examination, at least until the appearance of polyps, which in FAP negative can typically be later. The subjects not found with genetic alterations may be exempted from a regime of endoscopic surveillance. Therapy, in all its aspects, pharmacological, endoscopic and surgical, is difficult to outline and should, where possible because customized in various configurations of the disease, be addressed in an effort a) to prevent the formation and reduce the size and number of polyps, b) decontaminate a remove polyps, c) remove, together, all the diseased bowel, $\mathrm{d}$ ), treat, ontologically and radically, the disease when CRC has developed. It is hardly necessary to remind the big deal with the diagnosis and treatment of extracolonic injuries (or ECM). Of course many of these situations and these goals are often interrelated. Surgical options available are: the IRA, easier technical execution and with best functional results, that however is burdened by the need of endoscopic controls and removing of rectal stump polyps and the risk, unfortunately not be eliminated even with tight controls, of onset of colorectal cancer. The other surgical option is the most radical and extensive IPAA, burdened with a higher risk of postoperative complications and malfunctioned results, sometimes difficult to control. Genetic analysis can allow you to distinguish early risk patients (early onset) and late risk patients (late onset) and then choose the timing of surgical treatment, but the issue of what kind of treatment to choose, even if postponed, remains in any case. The examination test may also indicate patients at risk of developing extra-intestinal diseases, which requires an extension of the diagnostic procedures of screening also for this latter. These undeniable surgical problems have led to extend the possibilities of endoscopy in the programs of therapeutic combinations. As however endoscopists can engage in extensive treatments of polypectomy, the consideration of bleeding and perforating of these procedures, certainly not negligible, and the consideration that a polypectomy does not control the pathological evolution of polyps' basis on the wall of the bowel, make clear the limits inherent in applying extensive and / or routine procedure. Moreover, we must remember that not only pre-operatively, but even after surgery, the endoscopic control should be extended to the surveillance of the rectal stump, not only - as it is clear - after IRA, but also after IPAA since with the use of automatic staplers it is not rare that it remains a few inches of the rectal wall or even that the mucosectomy can in some cases leave mucous remnants $[3,12,13]$. Not to mention the sporadic reports of polyps and carcinomas in the pouch of patients operated on. But in addition to colonoscopy, also gastro-duodeno-endoscopy find place to highlight extra-colic gastro duodenal tumors. The TAC must be used to reveal the desmoids tumors and other spread diseases of the syndromes of Garder and Turcot. Contrasting the expectations of drug treatment. Aspirin and its derivatives are considered to be able to reduce the size and the number of adenomatous polyps. The same result seem to get the oral supplementation of $\mathrm{Ca}++$, vitamin C, NSAIDs and cyclooxygenase inhibitors (Cox2), so that was conducted research programs on the use of these drugs in combination with endoscopic surveillance. If an association of this kind may be reasonable for AFAP and MYH, data from other research - that do not confirm the antipolyposic action of the drug in question - suggests limited interest in the adoption of these combined treatments in FAP $[1,10]$. In conclusion, as well as on the side of the screening, also with regard to treatment and surveillance of the disease, much is delegated to genetic test-. Given the complexity and costliness of diagnostic procedures, the genotypephenotype correlation can direct the use of various procedures to be used for the specific problems of the different mutations of codons of the APC gene. , these predictable diseases, possibly to be seized at their onset. With the repeat these diagnostic procedures, is necessary not to render intolerable the quality of life of patients is not to burden intolerably cost the NHS.

\section{Conclusions \\ Operational suggestions}

The treatment of the disease remains difficult to manage. From the data of the literature and those derived from our experience, it is possible to identify some practical suggestions. Remains first step the key role of genetic testing to address the temporary abstention, the timing and manner of surveillance treatment, either pharmacological or endoscopic or surgical, single or in combination, also in relation to the types of codons affected by the mutations. Then appears preferable, especially when all the investigations carried out point to an indication for surgical treatment, have a frank conversation with 
the patient so that it is informed of the positive and negative data of surgical methods proposed (IRA and IPAA), also choosing which solution to adopt on the basis of a conscious evaluation. In patients who face the problem of relational life and in full working, perhaps a compromise solution can be the IRA with conservation of about $8-10 \mathrm{~cm}$ of the rectum, keeping in mind that for the most aggressive solutions, in males, in addition to the problems of fecal continence, may enter the field abnormalities of urine function and especially of the sexual / reproductive functions. Perhaps the best prospects are related to gene therapy or to IFV. I fare not yet clinically possible attempts to correct genetic mutations (with inserting a non-mutated FAP gene, viruses carried, in the genome of the patient, before the phenotyping of disease), it is likely that with the evolution of the techniques of in vitro fertilization (IFV), you can envisage the pre-implantation genetic diagnosis (PGD) as a new methodology designed to identify, before implantation in the uterus, the presence of genetic diseases in the embryo created in vitro by couples at high risk of reproductive diseases genetically determined.

The problem are:

a) the great implications of the genetic correction, , for increasingly complex assessment decision

b) The ethical implications of the PDG especially the fate of discarded embryos, even considering the risk that the methods can become, extending other genetic characteristics, as a new form of eugenics practice.

One final observation remains to be done on the implications of "genetic counseling", which certainly can indicate the proper management of the timing of the monitoring and treatment, but not for the psychological effects on the patient and family, the interpretation of the results obtained from the tests genetic may not be correct, these impose a lot of caution and seek to manage information obtained without rigid extremists or easy enthusiasms. $\therefore$ So one must be cautious in adopting the new discoveries because if the scientific concepts can be corrected, their clinic applications in the individual patients risk becoming a problem

\section{References}

1. Cavallaro A, Lauretta A, Cavallaro M, Pennisi S, Cavallaro V (2006) Annal
Italiani di Chirurgia. [Surgery on gastrountestinal stromal tumor CD117+ (G.I.S.T.): personal experience]. Ann Ital Chir 77: 137-114.

2. Webb WA, McDaniel L, Jones L (1985) Experience with 1000 colonoscopic polypectomies. Ann Surg 201: 626-632.

3. Konishi F, Morson BC (1982) Pathology of colorectal adenomas: a colonoscopic survey .J Clin Pathol 35: 830-841.

4. Kozuka S (1975) Premalignancy of the mucosal polyp in the large intestine: I. Histologic gradation of the polyp on the basis of epithelial pseudostratification and glandular branching. Dis Colon Rectum 18: 483-493.

5. Dukes CE, Bussey HJR (2013) The spread of rectal cancer and its effect on prognosis. Br J Cancer 12: 309-320.

6. Al-Tassan N, Chmiel NH, Maynard J, Fleming N, Livingston AL, et al. (2002) Inherited variants of MYH associated with somatic G:C fi T:A mutations in colorectal tumors. Nat Genet 30: 227-232.

7. Jones S, Emmerson P, Maynard J, Best JM, Jordan S, et al. (2002) Biallelic germline mutations in $\mathrm{MYH}$ predispose to multiple colorectal adenoma and somatic G:C fi T:A mutations. Hum Mol Genet 11: 2961-2967.

8. Jones S, Lambert S, Williams GT, Best JM, Sampson JR, et al. (2004) Increased frequency of the k-ras G12C mutation in MYH polyposis colorectal adenomas. Br J Cancer 90: 1591-1593.

9. Borrelli A, Carlomagno N, Dodaro C, et al. (2003) "Ereditary Colorectal Cancer and Molecular Analisys", Tehniques in Coloproctology 7- Supp.2, 2003.

10. Giardiello FM, Yang VW, Hylind LM, Krush AJ, Petersen GM, et al. (2002) Primary chemoprevention of familial adenomatous polyposis with sulindac. $\mathrm{N}$ Engl J Med 346:1054-1059.

11. Tonelli F, Ficari F, Bargellini T, Valanzano R (2012) “lleal Pouch Adenomas and Carcinomas after ristorative Proctocolectomy for Familial Adenomatous Polyposis". Dis Colon Rectum 55: 322-329.

12. GRAZIANO A (2007) Risk factor of anastomotic complication in rectal cancer POLICLINICO. SEZIONE CHIRURGICA114: 3-11.

13. Cavallaro A, Lauretta A, Cavallaro M, Pennisi S, Cavallaro V (2006) Surgery on gastrountestinal stromal tumor CD117+ (G.I.S.T.): Personal experience. [La chirurgia nei tumori stromali gastrointestinali. Ann Ital Chir 77: 137-141.

14. Hernegger GS, Moore HG, Guillem JG (2002) Attenuated familial adenomatous polyposis: an evolving and poorly understood entity. Dis Colon Rectum 45: 127-134.

15. Concetta Dodaro (2010) Attualità in tema di poliposi colica familiare. Dpt. di Scienze biomediche avanzate. UniversitàFederico II. 2010, Napoli.

16. GRAZIANO A (2007) J pouch colon anal reconstruction after anterior resection for cancer ACTA CHIRURGICA MEDITERRANEA 23: 97-100.

Copyright: (c) 2016 Paolo Graziano GM, et al. This is an open-access article distributed under the terms of the Creative Commons Attribution License, which permits unrestricted use, distribution, and reproduction in any medium, provided the original author and source are credited. 